Research Article

\title{
Optimal Location and Sizing of UPQC in Distribution Networks Using Differential Evolution Algorithm
}

\author{
Seyed Abbas Taher and Seyed Ahmadreza Afsari \\ Department of Electrical Engineering, Faculty of Engineering, University of Kashan, \\ Kashan 87317-51167, Iran \\ Correspondence should be addressed to Seyed Abbas Taher, sataher@kashanu.ac.ir
}

Received 26 January 2012; Revised 14 June 2012; Accepted 29 June 2012

Academic Editor: Hung Nguyen-Xuan

Copyright (C) 2012 S. A. Taher and S. A. Afsari. This is an open access article distributed under the Creative Commons Attribution License, which permits unrestricted use, distribution, and reproduction in any medium, provided the original work is properly cited.

\begin{abstract}
Differential evolution (DE) algorithm is used to determine optimal location of unified power quality conditioner (UPQC) considering its size in the radial distribution systems. The problem is formulated to find the optimum location of UPQC based on an objective function (OF) defined for improving of voltage and current profiles, reducing power loss and minimizing the investment costs considering the OF's weighting factors. Hence, a steady-state model of UPQC is derived to set in forward/backward sweep load flow. Studies are performed on two IEEE 33-bus and 69-bus standard distribution networks. Accuracy was evaluated by reapplying the procedures using both genetic (GA) and immune algorithms (IA). Comparative results indicate that DE is capable of offering a nearer global optimal in minimizing the OF and reaching all the desired conditions than GA and IA.
\end{abstract}

\section{Introduction}

Power quality and maintaining voltage magnitude at an acceptable range are gaining significant attention these days as an increasing range of equipments, sensitive to distortions or dips, are used in supply voltages $[1,2]$. Modern techniques and power electronic devices such as FACTS have improved considerably the power quality. Custom power devices as a part of FACTS devices are increasingly being used in custom power applications for improving power quality of power distribution systems such as SSTS (Solid State Transfer Switch), DVR (Dynamic Voltage Restorer), DSTATCOM (Distribution Static Compensator), and UPQC (Unified Power Quality Conditioner) [3-5].

Parallel connected converters can also improve current quality, while the series connected regulators might be employed to improve voltage quality [6]. As an effective approach, 
UPQC can function both as DSTATCOM and DVR as shunt and series compensators, respectively [7-10].

The UPQC consists of two voltage source inverters that are connected to a DC energy storage capacitor, to be used for improving voltage sag, unbalance, and flicker, as well as harmonics, dynamic active and reactive power regulation [11-14]. The series part inserts voltage in order to maintain it balanced and free of distortion, at the point of common coupling (PCC). Simultaneously, UPQC shunt part, injects current to the PCC in such a way that the entering current to the PCC bus is balanced sinusoidally.

Most UPQC studies deal with two bus distribution systems and consider UPQC behavior, dynamically in a short duration and not in long terms [8, 13, 15-19]. UPQC theory and modeling are described previously [20], while its topology and control, used simultaneously in voltage or current control mode, are presented in [1]. In another work, UPQC is applied in an experimental system with a control strategy $[8,15]$ having focused on the flow of instantaneous active and reactive power inside the UPQC. A new connection for UPQC to improve the power quality of two feeders in a distribution system is described in [7]. In the present study, similar to [21,22], a suitable model of UPQC in load flow calculation is proposed for steady state voltage compensation.

Differential evolution (DE) algorithm, considered as one of the best evolutionary algorithms, is widely used to solve optimization problems in general [23, 24]. DE algorithm is a parallel direct search method for generating trial parameter vectors and is used for minimizing objective function. It requires few control variables, is robust, easy to use, and lends itself very well to parallel computation.

In this paper, a new approach is applied using DE to determine optimal location and sizing of an UPQC in distribution networks in order to reduce power and energy losses, improve voltage profile, decrease lines currents, and minimize installation cost of UPQC. The amount of series and shunt reactive power, which is exchanged by UPQC in order to compensate voltage of PCC to a desired value, is derived by phasor model and correlated equations. Results in this work indicate superiority of DE over GA and IA methods as it converges faster and presents more certainty than both GA and IA.

\section{UPQC Structure and Modeling in Distribution Load Flow}

\subsection{UPQC Structure}

UPQC system configuration includes a combination of a series and shunt active filters [25] as presented in Figure 1.

In this work, effect of UPQC on voltage regulation of predetermined load (bus) in a steady-state power system is studied assuming that no active power is exchanged between UPQC and the system $[14,21,26,27]$.

\subsection{Modeling of UPQC in the Distribution Load Flow}

Forward/backward sweep load flow calculations are used in conjunction with a suitable steady state model for UPQC as presented by [28]. A section of a sample distribution network is shown in Figure $2[29,30]$ assuming that the 3-phase radial distribution network is in balance. Impedance between bus $i$ and bus $i+1$ is shown with $R_{i}+j X_{i}$. Local loads are connected in bus $i$ and bus $i+1$ (named $P_{i}+j Q_{i}$ and $P_{i+1}+j Q_{i+1}$ ) with their voltages being $V_{i}$ and $V_{i+1}$, 


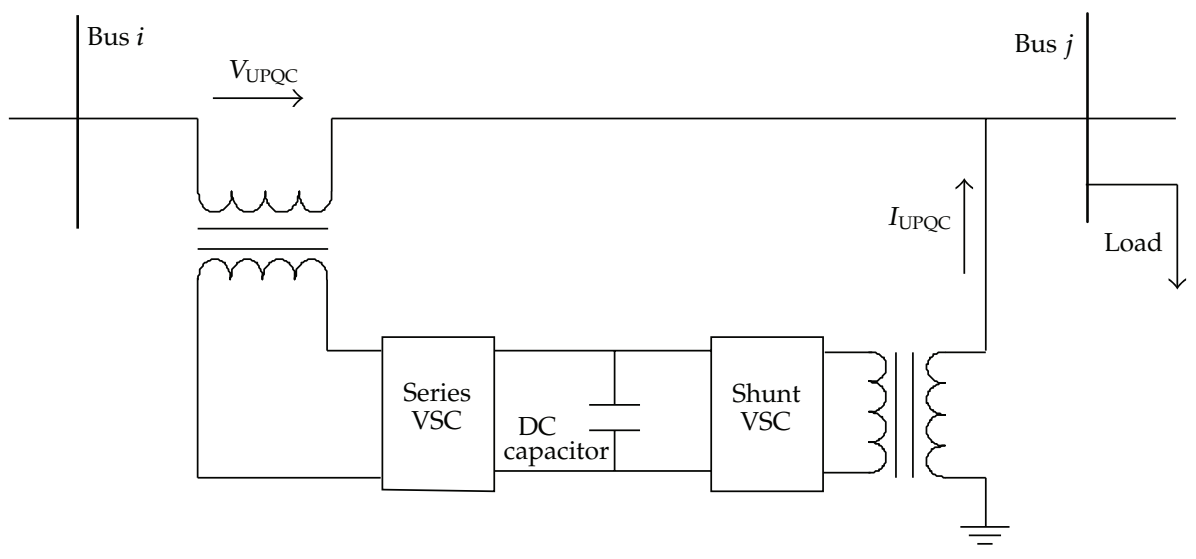

Figure 1: A typical UPQC structure.

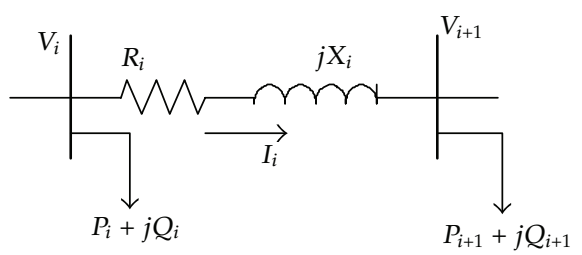

Figure 2: Single-line diagram of two consecutive buses of a distribution system.

respectively. The corresponding voltage phasor diagram in Figure 2 is presented in Figure 3 and the associated KVL equation is

$$
V_{i+1} \angle \theta_{i+1}=V_{i} \angle \theta_{i}-\left(R_{i}+j X_{i}\right) I_{i} \angle \delta
$$

Values of the variables are derived from the load flow. Usually in a network, the buses voltage is less than $1 \mathrm{pu}$, in which case, one can assume that voltage of bus $i+1$ is also less than 1 pu.

It is assumed that voltage magnitude of bus $i+1$ is compensated to a desired value (e.g., $1 \mathrm{pu}$ ). Hence, in a steady-state condition, the new angle of compensated voltage, injected reactive power, and voltage of the series compensator as well as injected reactive power and current of the shunt compensator can be calculated.

As shown in Figure 4, an UPQC is installed in bus $i+1$ in order to compensate voltage of bus $i+1$ to a desired value. $V_{\text {se }}$ must be kept perpendicular to the series compensator current. $I_{\mathrm{se}}$ and $I_{\mathrm{sh}}$ must also be kept perpendicular to the $V_{i+1}^{\prime}$.

For simplicity, $\angle V_{i+1}$ in phasor diagram is assumed to be zero. Hence,

$$
V_{i+1}^{\prime} \angle \theta_{i+1}^{\prime}=\vec{V}_{\mathrm{se}}+V_{i}^{\prime} \angle \theta_{i}^{\prime}-\left(R_{i}+j X_{i}\right)\left(I_{i} \angle \delta+I_{\mathrm{sh}} \angle\left(\frac{\pi}{2}+\theta_{i+1}^{\prime}\right)\right),
$$




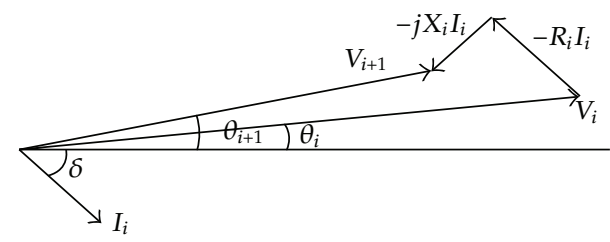

Figure 3: Phasor diagram of voltages and current of system shown in Figure 2.

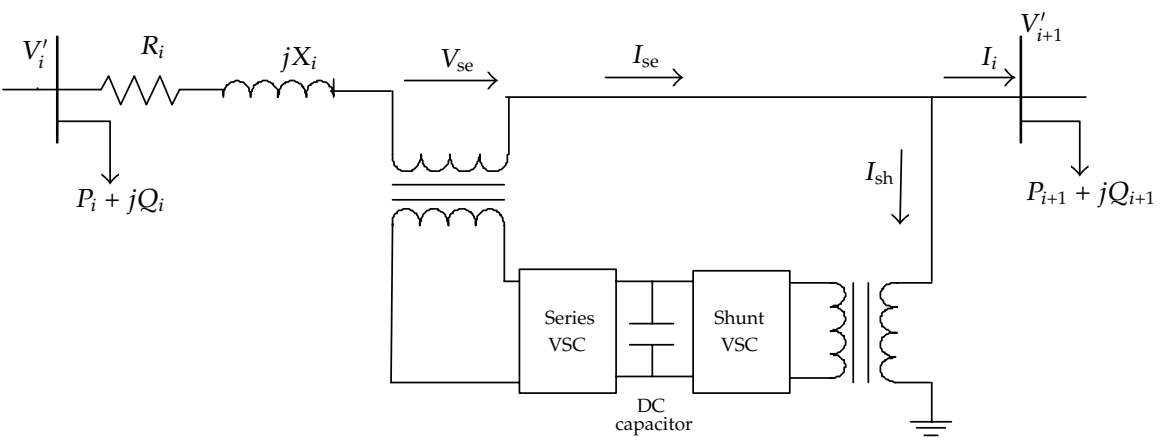

Figure 4: Installing an UPQC in distribution system.

where (') denotes the amount of variable after compensation,

$$
\begin{aligned}
\angle I_{\mathrm{sh}} & =\frac{\pi}{2}+\theta_{i+1}^{\prime}, \\
\vec{I}_{\mathrm{se}} & =\vec{I}_{i}+\vec{I}_{\mathrm{sh}} .
\end{aligned}
$$

$V_{i}^{\prime} \angle \theta_{i}^{\prime}$ and $I_{i} \angle \delta$ may be calculated by the load flow equations. $\angle I_{\text {se }}$ might present itself in two states; firstly, $\vec{I}_{\text {se }}$ may have a lagging angle in which case $\angle V_{\text {se }}$ can be calculated using (2.4) and the corresponding phasor diagram may be expressed as Figure 5 below:

$$
\angle V_{\mathrm{se}}=\angle I_{\mathrm{se}(\mathrm{lag})}+\frac{\pi}{2}
$$

Secondly, $\vec{I}_{\mathrm{se}}$ has a leading angle where $\angle V_{\text {se }}$ is expressed by (2.5) with the corresponding phasor diagram as shown in Figure 6.

Separating real and imaginary parts of (2.2) into two equations allows its solution despite $I_{\mathrm{sh}}, \theta_{i+1}^{\prime}$, and $V_{\mathrm{se}}$ being unknown quantities. Here, it is assumed that the reactive power injected by the shunt part of UPQC $\left(Q_{\mathrm{sh}}\right)$ is modeled as a negative constant reactive load in bus $i+1$ (see Figures 7, 8, and 9), thereby allowing $\theta_{i+1}^{\prime}$ and $\vec{V}_{\text {se }}$ to be determined:

$$
V_{\mathrm{se}} \angle \alpha=V_{i+1}^{\prime} \angle \theta_{i+1}^{\prime}+\left(R_{i}+j X_{i}\right)\left(I_{i}^{\prime} \angle \delta\right)-V_{i} \angle \theta_{i}
$$

Following some computations and rearrangements, (2.6) represents the separated real and imaginary parts of (2.5):

$$
\begin{aligned}
& a_{1} x_{1}=b \cos x_{2}+c_{1}, \\
& a_{2} x_{1}=b \sin x_{2}+c_{2},
\end{aligned}
$$




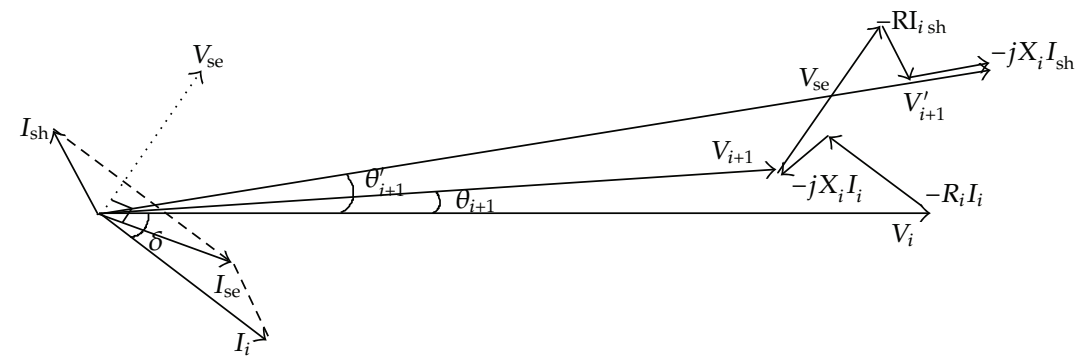

Figure 5: Phasor diagram of voltage and current of system shown in Figure 4 in lagging mode of $I_{\text {se }}$.

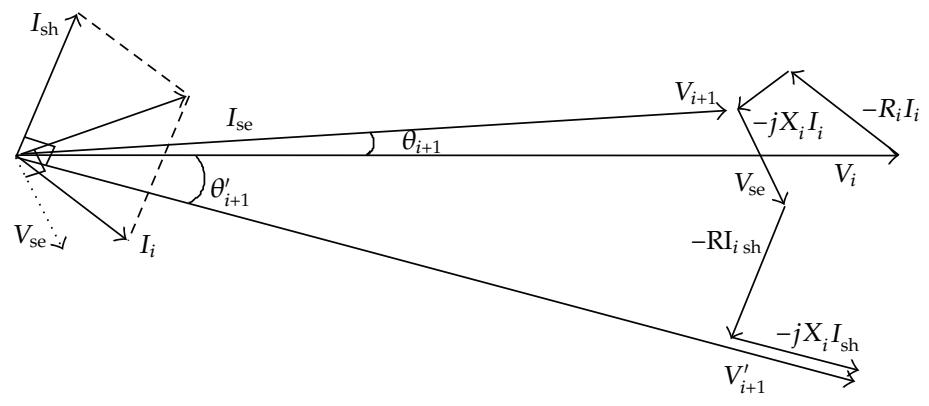

Figure 6: Phasor diagram of voltage and current of system shown in Figure 4 in leading mode of $I_{\mathrm{se}}$.

where

$$
\begin{gathered}
a_{1}=\cos \rho, \quad a_{2}=\sin \rho, \quad b=V_{i+1}^{\prime}, \\
c_{1}=\operatorname{real}\left(\left(R_{i}+j X_{i}\right)\left(I_{i \text { new }} \angle \beta\right)\right)-\operatorname{real}\left(V_{i} \angle \theta_{i}\right), \\
c_{2}=\operatorname{imag}\left(\left(R_{i}+j X_{i}\right)\left(I_{i \text { new }} \angle \beta\right)\right)-\operatorname{imag}\left(V_{i} \angle \theta_{i}\right), \\
x_{1}=V_{\text {se }}, \quad x_{2}=\theta_{i+1}^{\prime}, \\
x_{1}=\frac{-B \pm \sqrt{\Delta}}{2 A}, \quad \Delta=B^{2}-4 A C, \\
x_{2}=\cos ^{-1}\left(\frac{a_{1} x_{1}-c_{1}}{b}\right)=\sin ^{-1}\left(\frac{a_{2} x_{1}-c_{2}}{b}\right) .
\end{gathered}
$$

Here, $A, B$, and $C$ are defined as

$$
A=\frac{a_{1}^{2}+a_{2}^{2}}{b^{2}}, \quad B=-2 \frac{a_{1} c_{1}+a_{2} c_{2}}{b^{2}}, \quad C=\frac{c_{1}^{2}+c_{2}^{2}}{b^{2}} .
$$

As can be seen from (2.11), two roots might be assigned for the variable $x_{1}$, and therefore, two values can be obtained for $\theta_{i+1}^{\prime}$. In order to verify the correct answers, the following corresponding boundary conditions need to be examined:

$$
b=V_{i+1}^{\prime}=V_{i+1} \longrightarrow V_{\mathrm{se}}=0 .
$$




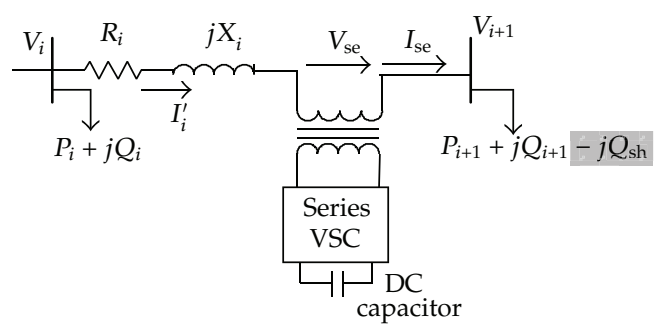

Figure 7: Installing an UPQC in a distribution system by modeling shunt compensator as constant reactive load.

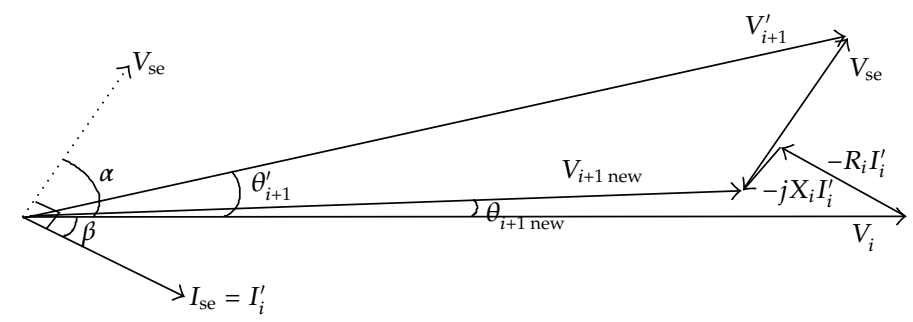

Figure 8: Phasor diagram of voltage and current of system that shown in Figure 7 in lagging mode of $I_{\mathrm{se}}$.

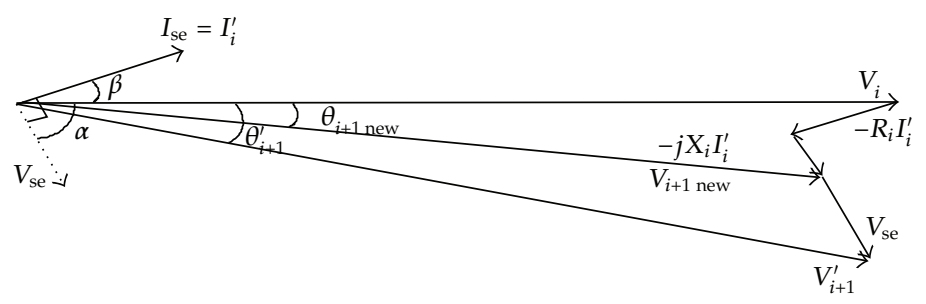

Figure 9: Phasor diagram of voltage and current of system shown in Figure 7 in leading mode of $I_{\text {se }}$.

$x_{1}=(-B+\sqrt{ } \Delta) / 2 A$ is found to be the correct answer in (2.11). Hence, the reactive power injected to the network by the series part of UPQC $\left(Q_{\text {se }}\right)$ for voltage correction of the connected bus to $V_{i+1}^{\prime}$, may be expressed as

$$
j Q_{\mathrm{se}}=\vec{V}_{\mathrm{se}} \cdot \vec{I}_{i}^{*}
$$

When $Q_{\text {se }}$ is greater than its maximum limit $\left(Q_{\text {se }_{\max }}\right)$, it can be derived by $Q_{\text {se }_{\max }}$.

However, voltage magnitude of the compensated bus cannot be regulated in the desired value. Thus, a new voltage magnitude $\left(V_{i+1 \text { new }}^{\prime}\right)$ and a new phase angle $\left(\theta_{i+1 \text { new }}^{\prime}\right)$ of compensated bus may be expressed as

$$
V_{i+1_{\max }}^{\prime} \angle \theta_{i+1_{\max }}^{\prime}=V_{i} \angle \theta_{i}-\left(\left(R_{i}+j X_{i}\right) I_{i_{\text {new }}} \angle \beta\right)+V_{\text {se }_{\max }} \angle \alpha .
$$

\subsection{Installing Model in Load Flow}

In order to evaluate load flow at steady-state conditions, in the presence of UPQC in a load flow, voltage magnitude of the compensated bus $(i+1)$ can be assumed to be any desired value 
(with any iteration in the forward sweep), and therefore, the amount of injected reactive power by shunt part of UPQC, $\left(Q_{\text {sh }}\right)$ may be modeled as a negative constant load. At this stage, the phase angle of the compensated voltage and amount of injected reactive power produced by $Q_{\text {se }}$ can be calculated using the above equations [21].

The boundary value of $Q_{\text {se }}$ should be examined. In case it was greater than maximum rating limit, magnitude and phase angle of the compensated bus are calculated using (2.16), having $Q_{\text {se }}$ set to its maximum rating. Now new magnitude and phase angle of the compensated bus are used to determine voltages for buses located downstream to the compensated bus. At this stage, new updated voltages of buses and $Q_{\text {sh }}$ can be used to calculate load currents in the backward sweep. This procedure is repeated until load flow convergence reaches the desired tolerance.

\section{Problem Formulation}

In this study, optimal location and sizing of UPQC in a steady state condition are obtained to improve power quality. Hence, minimizing UPQC size and power loss in the distribution network, is considered as the objective function (OF). The voltage and current constraints are formulated as a penalty function to the $\mathrm{OF}$.

\subsection{Objective Function}

Equation (3.1) illustrates mathematically the proposed OF as below:

$$
\mathrm{OF}=\left[K_{e} \sum_{i=1}^{3}\left(T_{i} \times P_{\text {oss }_{i}}\right)+\sum_{i=1}^{3}\left(K_{c i} \times \operatorname{Cost}_{\mathrm{UPQC}_{\mathrm{year}_{i}}}\right)\right] \times\left[\prod_{i=1}^{3}\left|\left(\prod_{j=1}^{n l} \mathrm{OC} \times \prod_{j=1}^{n b} \mathrm{OV}\right)\right|\right],
$$

where $i, n b$, and $n l$ indicate numbers of load level, bus, and lines, respectively; $K_{e}$ is the energy cost of losses, $T_{i}$ is the time duration of $i$ th load level, and $K_{c i}$ is the time duration proportion of $i$ th load level to the total time duration [31], determined as

$$
K_{c i}=\frac{T_{i}}{\sum_{i=1}^{3} T_{i}}
$$

$P_{\text {loss }_{i}}$ is the total power loss in the $i$ th load level, described [32] as

$$
P_{\text {oss }_{i}}=\sum_{j=1}^{n l} R_{j}\left|I_{j}\right|^{2}
$$

The first term in the OF equation above corresponds to the total costs (in US \$) of power loss and UPQC installation which should be minimized. The second term deals with the voltages and currents limitations of network which is an important factor to be bonded within the desired limits. This term acts as a penalty factor and is assigned in a constant ratio to the first term, in response to the deviation from specific boundary conditions, and equals to 1 when all limitations are secured for buses voltages and lines currents. 


\subsection{Cost of UPQC}

The cost of UPQC is assumed to be the same as the cost of UPFC as reported by Siemens database. Cost of investment can be determined from UPQC cost [33-35] as

$$
\begin{gathered}
\operatorname{Cost}_{\mathrm{UPQC}}(\mathrm{US}(\$ / \mathrm{kVAr}))=0.0003 S^{2}-0.2691 S+188.22, \\
\operatorname{Cost}_{\mathrm{UPQC}_{\text {year }_{i}}}=\operatorname{Cost}_{\mathrm{UPQC}} \frac{(1+B)^{n_{\mathrm{UPQC}}} \times B}{(1+B)^{n_{\mathrm{UPQC}}}-1} .
\end{gathered}
$$

In the above equations, $S$ is the operating range of the UPQC in MVAr, Cost $\mathrm{UPQC}_{i}$ is the investment cost for the $i$ th load level in the year of allocation, Cost $_{\mathrm{UPQC}_{\text {vear }}}$ corresponds to the annual cost of UPQC for the same load level, $n_{\mathrm{UPQC}}$ is the longevity of UPQC, and $B$ is the asset rate of return.

Minimizing the deviation of node's voltage and line's current is formulated in the second term of OF. OC and OV denote line over current factor and voltage stability index, respectively, and are defined [36] as

$$
\begin{gathered}
\mathrm{OC}= \begin{cases}1 ; & \text { if } I_{j} \leq I_{\max }, \\
\exp \left(\lambda\left|1-\frac{I_{j}}{I_{\max }}\right|\right) ; & \text { if } I_{j}>I_{\max },\end{cases} \\
\mathrm{OV}= \begin{cases}1 ; & \text { if } V_{\min } \leq V_{b} \leq V_{\max }, \\
\exp \left(\mu\left|1-V_{b}\right|\right) ; & \text { otherwise. }\end{cases}
\end{gathered}
$$

Here, $I_{j}$ is the current magnitude flow for the $j$ th line, $I_{\max }$ is the maximum current that can flow in the network lines, $\lambda$ and $\mu$ are small positive constants, and $V_{b}$ is the voltage magnitude for the $b$ th bus. If all line currents are less than $I_{\max }, \mathrm{OC}$ will be equal to 1 , and if all buses voltages are within the desired boundaries, OV would equal unity; in all other conditions, OC or OV will acquire a value (greater than 1) representing the penalty factor in OF.

Total cost saving (TCS) is the difference between total energy loss cost before installation, and the sum of annual cost of UPQC and total energy loss cost after installation in the three load levels (light, medium, and peak) considered here and may be expressed as

$$
\mathrm{TCS}=K_{e} \sum_{i=1}^{3} T_{i} \cdot P_{\text {loss }_{i}}-K_{e} \sum_{i=1}^{3} T_{i} \cdot P_{\text {loss }_{i}}^{\text {With UPQC }}-\sum_{i=1}^{3} K_{c i} \cdot \text { Cost }_{\mathrm{UPQC}_{\text {year }}} .
$$

\section{Differential Evolution Algorithm}

Amongst the best known direct search approaches for nonlinear, nondifferentiable objective function, introduced so far, differential evolution (DE) has proved to be an effective algorithm. Inherently parallel search techniques like genetic algorithms and evolution strategies have some built-in safeguards to forestall misconvergence [37]. DE algorithm is a stochastic, population-based optimization algorithm introduced by Storn and Price in 1997 [38]. It creates new candidates solutions by combining the parent individual and several other individuals of the same population. DE generates new vectors of parameter by adding the weighted 
difference between two population vectors to a third one [39]. A candidate replaces the parent only if it has better fitness value [40]. DE is an effective, fast, simple, robust, inherently parallel, and has few control parameters need little tuning. It can be used to minimize noncontinuous, nonlinear, and nondifferentiable space function, also it can work with noisy, flat, multidimensional, and time-dependent objective functions and constraint optimization in conjunction with penalty functions [39].

The main differences between genetic algorithm (GA), immune algorithm (IA) [41], and DE are the selection process and the mutation that makes DE self-adaptive [42]. A practical optimization technique is expected to fulfill three requirements: regardless of the initial system parameter values, the method should find the true global minimum; it should converge rapidly; should be easy to use, that is, it should possess limited number of control parameters [43-46]. The initial population of a DE algorithm is randomly generated within the control variable bounds. This population is successfully improved over generations by applying mutation, crossover and selection operators, to reach an optimal solution. The size of population, however, is constant during the process. At the end of each generation, the best individuals based on his OF value are stored. In short, DE operation includes four stages [24, 43-46] as described below.

\subsection{Population Initialization}

Initial population is a number of parameter vectors (PVs), randomly generated within the target parameters' limits. For the Gth generation, the population contains $N_{p}$ multidimensional PVs $\mathbf{x}_{\mathbf{i}, \mathrm{G}}=\left[x_{i, G^{\prime}}^{1} x_{i, G^{\prime}}^{2} \ldots, x_{i, G}^{D}\right]$, where $i$ represents the number of the PV. The $k$ th parameter in the $i$ th PV of the first generation can be obtained by (4.1):

$$
x_{i, 1}^{k}=x_{\min }^{k}+\operatorname{rand}(0,1) \times\left(x_{\max }^{k}-x_{\min }^{k}\right) \quad i \in\left[1, N_{p}\right], k \in[1, D],
$$

where $x_{\min }^{k}$ and $x_{\max }^{k}$ are the lower and upper bounds of the $k$ th parameter, respectively, and rand $(0,1)$ is a random scalar within $[0,1]$ as shown in Figure 10. In case there is a priori knowledge available about the problem, the preliminary solution may be included to the initial population by adding normally distributed random deviations to the nominal solution [47].

\subsection{Mutation}

DE does not use a predefined probability density function to generate perturbing fluctuations. It relies upon the population itself to perturb the vector parameter [48]. For each member $i$ from the population, DE generates a mutated PV, $v_{i, G+1}$, by adding the weighted difference of two randomly selected PVs to a third randomly selected PV as

$$
v_{i, G+1}=x_{r_{3}, G}+s\left(x_{r_{1}, G}-x_{r_{2}, G}\right),
$$

where the subscripts $r_{1}, r_{2}$, and $r_{3}$ represent the randomly selected PVs such that $r_{1} \neq r_{2} \neq r_{3} \neq i$. $s$ is a user-defined constant called the step size. It controls the scale of differential variation and usually selected to be in the range of $0 \leq s \leq 2$. The corresponding objective function will 


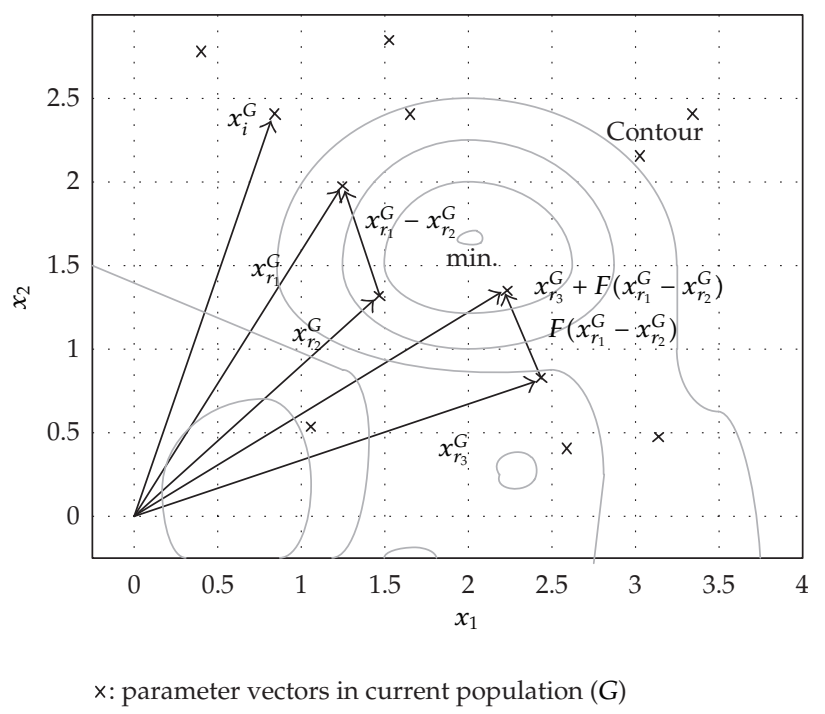

Figure 10: Two-dimensional example of DE method (creation of new generation from current generation).

be compared with a predetermined individual PV. Note that if any parameter of the mutated $\mathrm{PV}, v_{i, g}$, is found outside the related boundaries, it will be fixed at the corresponding upper or lower limits. This ensures that the best parameter vector is evaluated for every generation in order to track the progress made throughout the minimization process.

\subsection{Crossover Operation}

The main idea behind DE is a scheme for generating trial PVs. When faced with small population diversity, the population could rapidly cluster together leading to premature convergence and restricted improvement. In such circumstances, in order to increase the local diversity of the mutant populations, a crossover is introduced [48]. For this purpose, parameters of the mutated PV, $v_{i, G+1}$, are mixed with the so-called target PV, $x_{i, G}$, in order to form the trial $\mathrm{PV}, u_{i, G+1}$ as below

$$
u_{i, G+1}^{k}= \begin{cases}v_{i}^{k} & \text { if } \operatorname{rand}_{i}^{k} \leq \mathrm{CR} \text { or } k=J_{\mathrm{rand}}, \\ x_{i, G}^{k} & \text { if } \operatorname{rand}_{i}^{k}>\mathrm{CR} \text { and } k \neq J_{\mathrm{rand}}\end{cases}
$$

where the superscript $k$ indicates that the $k$ th component of the trial PV, $\operatorname{rand}_{i}^{k}$ is a random scalar so that $0 \leq \operatorname{rand}_{i}^{k} \leq 1$, and $J_{\text {rand }}$ is a randomly chosen integer so that $1 \leq J_{\text {rand }} \leq D$. $J_{\text {rand }}$ is chosen once for each vector and ensures that $u_{i, G+1}$ obtains at least one parameter from $v_{i, G+1}$. CR, the DE controlling parameter, is called crossover rate which is user-defined and usually within the range $0<\mathrm{CR}<1$.

\subsection{Evaluation and Selection}

After generating the trial PV, $u_{i, G+1}$, if the obtained cost function is lower than the target PV, $x_{i, G}$, then $x_{i, G+1}$ will be set to $u_{i, G+1}$; otherwise $x_{i, G}$ will be retained. To complete an iteration, 


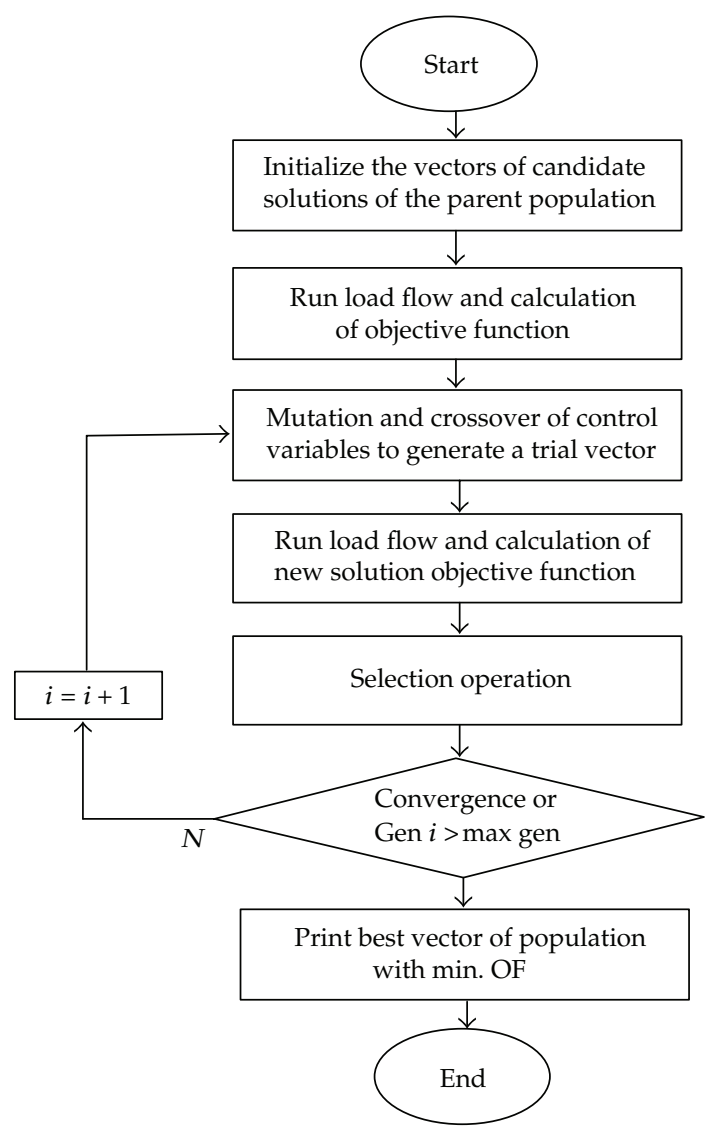

Figure 11: Flowchart of DE algorithm.

each PV of the population has to serve once as the target PV. All members of population with similar opportunity can be selected as parents. If parents have a better OF, they will be retained. The best of these are selected to reconstruct the new generation.

The procedure comes to a halt when an acceptable solution candidate is reached or no improvement in new generations is accrued or the number of iterations exceeds its limit. In Figure 11, a flowchart of DE algorithm is presented.

\section{Implementation of DE for Finding Optimal Location and Size of UPQC}

As mentioned before, DE algorithm is applied to search the best location and size of UPQC in network for each load level, such that OF becomes minimum, that means minimum power loss, size and cost of UPQC, and deviation of voltages/currents from the desired values.

Two case studies are presented in this section, including a 33-bus and 69-bus standard IEEE distribution network that work at $12.66 \mathrm{kV}$ and have radial structure $[49,50]$. These distribution networks are shown in Figures 12 and 13, respectively. 


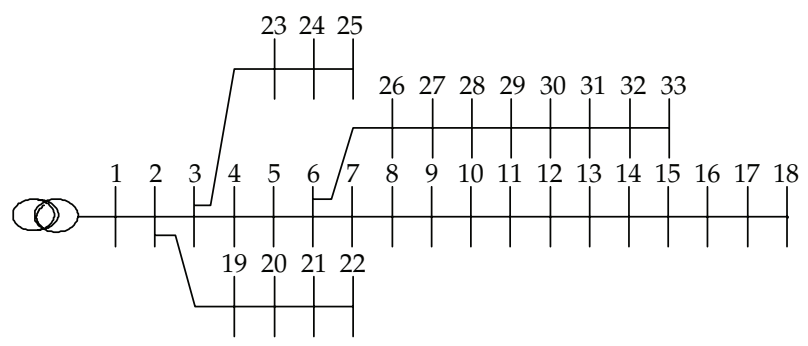

Figure 12: Single-line diagram of IEEE 33-bus distribution system.

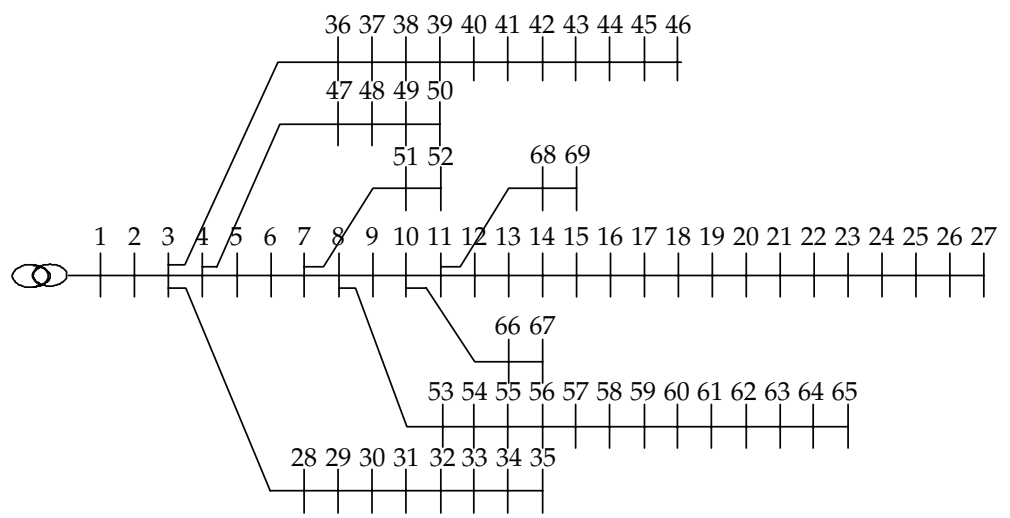

Figure 13: Single-line diagram of IEEE 69-bus distribution system.

In order to model the annual load profile, three load levels are selected including light, medium, and peak. Table 1 shows the time duration and total load for each load level in two test systems.

In order to evaluate the effectiveness of $\mathrm{DE}$, its performance is compared with GA and IA, run on the same basis. To find the optimum set of parameters for DE, GA, and IA, 50 trials are performed for each possible set of parameters. For each trial the optimum OF is recorded, and the appropriate statistical measures are compared in the following.

Maximum iteration and initial population sizes are set to 100 and 50, respectively. Initial binary strings (chromosomes) are randomly produced, containing a number of selected bus for compensation as well as three values for voltages of compensated node in three load levels. The corresponding DE, GA, and IA settings are shown in Table 2.

After running the load flow in three load levels, OF is calculated for each chromosome. The OF parameters applied are indicated in Table 3 [50-53].

It should be noted that the constraint of $Q_{\mathrm{UPQC}}$, voltages of buses, and currents of lines (for Lynx conductor [54] at $30^{\circ} \mathrm{C}$ ) are as indicated below:

$$
\begin{gathered}
0 \leq Q_{\mathrm{UPQC}}{ }^{\mathrm{kVAr}} \leq 10000^{\mathrm{kVAr}}, \\
0.9 \leq V_{\text {pu. }} \leq 1.1, \\
I_{\text {Max }} \leq 520^{A} .
\end{gathered}
$$


Table 1: Load level and load duration time in 33- and 69-bus IEEE test systems.

\begin{tabular}{lcccc}
\hline Load level & & Light & Medium & Peak \\
\hline Time duration $(\mathrm{h})$ & & 2000 & 5260 & 1500 \\
Total load (kVAr) & 33 buses & $3715+j 2300$ & $4829.5+j 2300$ & $5944+j 2300$ \\
& 69 buses & $3802.2+j 2694.6$ & $4942.8+j 2694.6$ & $6083.5+j 2694.6$ \\
Total power loss $(\mathrm{kW})$ & 33 buses & 202.68 & 305.86 & 442.41 \\
& 69 buses & 225.00 & 342.99 & 502.52 \\
\hline
\end{tabular}

Table 2: Parameters setting of the DE, GA, and IA methods.

\begin{tabular}{|c|c|c|c|c|c|c|c|}
\hline & $S$ & $\mathrm{CR}$ & Mutation rate & Selection rate & $P_{r}$ & $P_{c}$ & $P_{m}$ \\
\hline $\mathrm{DE}$ & 0.7 & 1 & - & - & - & - & - \\
\hline GA & - & - & 0.3 & 0.5 & - & - & - \\
\hline IA & - & - & 一 & - & 0.3 & 0.9 & 0.1 \\
\hline
\end{tabular}

Table 3: OF parameters setting for optimization.

\begin{tabular}{lccccccc}
\hline$n_{\mathrm{UPQC}}($ year $)$ & $B$ & $K_{e}$ US $(\$ / \mathrm{kWhr})$ & $\lambda$ & $\mu$ & \multicolumn{2}{c}{$K_{c i}$} \\
\hline 30 & & & & \multicolumn{3}{c}{ Load Level $(i)$} \\
& 0.1 & 0.06 & 2 & 1 & 1 & 2 & 3 \\
& & & & & 0.22831 & 0.60046 & 0.17123 \\
\hline
\end{tabular}

\section{Simulation Results}

The effectiveness of proposed approach is illustrated using IEEE 33-bus and IEEE 69-bus systems. It is tried to obtain the optimal solution using the OF given by (3.1) in two sample networks; the simulation results are described in the following sections.

\subsection{IEEE 33-Bus Test System}

Table 4 shows the placement and size of UPQC, and also the minimum OF using GA, IA, and DE methods in IEEE 33-bus distribution system in three load levels.

Compared with GA and IA, DE seems to offer an improved optimal solution with its lower OF. In this system, the 29th bus is selected for UPQC installation by DE and 33th and 28th bus are selected by GA and IA methods for compensating for light, medium, and peak load levels, respectively.

Although IA has managed to find installation location fairly close to that of the DE, it has performed weaker in finding the best size of UPQC in the three load levels. Location candidates have a discrete search space, while search space for size of UPQC is wide and continuous causing more difficult search for the IA method compared with DE. In this regard, GA does not seem to present any suitable result for both location and size as compared with DE. By increasing the load level, all algorithms suggest reasonably greater size of UPQC (with greater shunt size compared with series size). Table 5 presents comparison of power loss, annual cost of UPQC, number of under voltage buses, and number of over current lines before and after installation using DE algorithm in the three load levels, in the IEEE 33-bus distribution system. 
Table 4: Comparison results of OF value, optimal location, and sizing of UPQC in IEEE 33-bus test system.

\begin{tabular}{|c|c|c|c|c|c|}
\hline \multirow{3}{*}{ OF } & \multicolumn{2}{|l|}{$\mathrm{DE}$} & \multicolumn{3}{|c|}{158784.1} \\
\hline & \multicolumn{2}{|l|}{ GA } & \multicolumn{3}{|c|}{186510.3} \\
\hline & IA & & \multicolumn{3}{|c|}{165598.1} \\
\hline \multirow{4}{*}{ Location } & \multicolumn{2}{|l|}{$\mathrm{DE}$} & \multicolumn{3}{|c|}{29} \\
\hline & GA & & \multicolumn{3}{|c|}{33} \\
\hline & \multicolumn{2}{|l|}{ IA } & \multicolumn{3}{|c|}{28} \\
\hline & & & Light & Medium & Peak \\
\hline \multirow{6}{*}{ Size (kVAr) } & \multirow{2}{*}{$\mathrm{DE}$} & Shunt & 914.1 & 1158.1 & 2457.3 \\
\hline & & Series & 0.0015 & 39.6 & 125.9 \\
\hline & \multirow{2}{*}{ GA } & Shunt & 917 & 1574 & 2953.9 \\
\hline & & Series & 3.65 & 17.65 & 50.9 \\
\hline & \multirow{2}{*}{ IA } & Shunt & 508.1 & 910.6 & 1502 \\
\hline & & Series & 0.902 & 20.1 & 76.46 \\
\hline
\end{tabular}

Table 5: Summary results of IEEE 33-bus distribution system.

\begin{tabular}{lcccc}
\hline Load level & & Light & Medium & Peak \\
\hline \multirow{2}{*}{ Total power loss $(\mathrm{kW})$} & ${ }^{*}$ B I & 202.68 & 305.86 & 442.41 \\
& ${ }^{* *}$ A I & 150.3 & 244.44 & 402.7 \\
\hline Cost UPQCyear $(\$)$ & A I & 18254 & 23912 & 51575 \\
\hline \multirow{2}{*}{ Number of under voltage buses } & B I & 0 & 7 & 14 \\
\hline \multirow{2}{*}{ Number of over current lines } & A I & 0 & 0 & 0 \\
\hline
\end{tabular}

*Before installation.

${ }^{* *}$ After installation.

Table 6: Comparison of annual costs of IEEE 33-bus test system.

\begin{tabular}{ll}
\hline Total energy loss cost BI (\$) & $1.6067^{*} 10^{5}$ \\
Total energy loss cost AI (\$) & $1.3143^{*} 10^{5}$ \\
Total annual cost of UPQC $(\$)$ & $2.7357^{*} 10^{4}$ \\
Total cost saving $(\$)$ & $1.8829^{*} 10^{3}$ \\
\hline
\end{tabular}

Table 7: CPU time for optimization in IEEE 33-bus test system.

\begin{tabular}{lccc}
\hline Algorithm & DE & GA & IA \\
\hline CPU time (second) & 5512 & 9053 & 8102 \\
\hline
\end{tabular}

Results indicate a power loss reduction in all three load levels, and in general, the total power loss is reduced by $18.2 \%$, while all voltages and currents are within the desired limits. In the third load level, total power loss is decreased by $8.9 \%$ because size reduction of UPQC has led to buses voltages to go out of the desired boundaries and increased size of UPQC has led to increased power loss. Hence, this solution is an optimum point to supply both boundaries.

Table 6 presents the annual results of economic evaluations. As can be seen, total cost saving will be 1885.9 \$. 
Table 8: Comparison results of OF value, optimal location, and sizing of UPQC in IEEE 69-bus test system.

\begin{tabular}{|c|c|c|c|c|c|}
\hline \multirow{3}{*}{$\mathrm{OF}$} & \multicolumn{2}{|l|}{$\mathrm{DE}$} & \multicolumn{3}{|c|}{170181.9} \\
\hline & \multicolumn{2}{|l|}{ GA } & \multicolumn{3}{|c|}{220877.4} \\
\hline & IA & & \multicolumn{3}{|c|}{181761.2} \\
\hline \multirow{4}{*}{ Location } & \multicolumn{2}{|l|}{$\mathrm{DE}$} & \multicolumn{3}{|c|}{62} \\
\hline & & & \multicolumn{3}{|c|}{58} \\
\hline & \multicolumn{2}{|l|}{ IA } & \multicolumn{3}{|c|}{60} \\
\hline & & & Light & Medium & Peak \\
\hline \multirow{6}{*}{ Series size (kVAr) } & \multirow{2}{*}{$\mathrm{DE}$} & Shunt & 1089.7 & 1156.2 & 2240.2 \\
\hline & & Series & 11.3 & 73.8 & 280.6 \\
\hline & \multirow{2}{*}{ GA } & Shunt & 1200.3 & 1431 & 2290.3 \\
\hline & & Series & 24 & 111 & 312.1 \\
\hline & \multirow{2}{*}{ IA } & Shunt & 1092.8 & 1187.1 & 2235.9 \\
\hline & & Series & 32.6 & 71 & 300 \\
\hline
\end{tabular}

Table 9: Summary results of IEEE 69-bus distribution system.

\begin{tabular}{lcccc}
\hline Load level & & Light & Medium & Peak \\
\hline \multirow{2}{*}{ Total power loss $(\mathrm{kW})$} & B I & 225 & 343 & 502.5 \\
& A I & 155.1 & 265.7 & 437.1 \\
\hline CostuPQC year $(\$)$ & A I & 21948 & 23115 & 50150 \\
\hline \multirow{2}{*}{ Number of under voltage buses } & B I & 0 & 6 & 8 \\
& A I & 0 & 0 & 0 \\
\hline \multirow{2}{*}{ Number of over current lines } & B I & 0 & 0 & 4 \\
& A I & 0 & 0 & 0 \\
\hline
\end{tabular}

Table 10: Comparison of annual costs of IEEE 69-bus test system.

Total energy loss cost BI (\$)

Table 11: CPU time for optimization in IEEE 69-bus test system.

\begin{tabular}{lccc}
\hline Algorithm & DE & GA & IA \\
\hline CPU time (second) & 8137 & 13137 & 11433 \\
\hline
\end{tabular}

The proposed method has been implemented on a quad computer with $2.8 \mathrm{GHZ}$ CPU. Table 7 shows CPU time for each algorithm, indicating a much faster convergence by DE than GA and IA.

Figure 14 shows voltages of buses before and after UPQC installation using DE algorithm in each load level in the IEEE 33-bus distribution network. Results shows improve ment in voltage profiles after installation UPQC in each load level. Location of UPQC is indicated by the arrow.

The convergence curves for the OF obtained by DE, IA, and GA are represented in Figure 15. Results show that DE has a faster convergence and better ability in searching the 


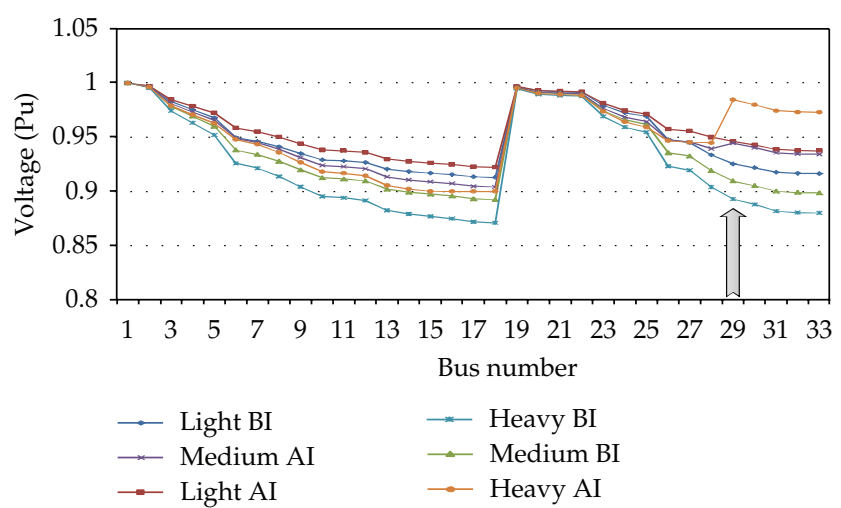

Figure 14: Voltages for the 33-bus distribution network before and after UPQC installation.

minimum OF compared with both IA and GA methods. DE is converged at the 44th iteration, while IA and GA have done so at the 53th and 65th iterations, respectively.

\subsection{IEEE 69-Bus Test System}

Table 8 shows placement and size of the UPQC as well as the minimum OF using DE, GA, and IA methods in the IEEE 69-bus system in the three load levels.

Similarly, DE offers better optimal solution here (i.e., lower OF and lower UPQC size) compared to GA and IA. In this 69-bus case study, the 62th bus is selected for UPQC installation by DE and the 58th and 60th buses are selected for UPQC installation by GA and IA methods, respectively, in the three load levels. Table 9 shows the similar results to those of Table 5, but for the 69-bus distribution system. Minimum voltage and maximum current of the system have improved and the system losses are reduced in each load level. Generally, total power loss was reduced by $21.42 \%$.

Annual results of economic evaluation for the 69-bus system are presented in Table 10 with total cost saving of 10288 \$. Table 11 shows CPU time for each algorithm. The same as 33 bus system, here, DE has a faster convergence and better optimal solution than both GA and IA.

Figure 16 shows voltages of buses before and after installation UPQC using DE algorithm in each load level for IEEE 69-bus distribution network. In this figure, improvement in voltage profile is evident. Location of UPQC is again represented by the arrow. Figure 17 shows the convergence diagram for DE, IA, and GA. Results show that DE has faster convergence compared with IA and GA so that DE converged at the 23th iteration, while IA and GA do it at the 55th and 65th iterations, respectively. Best candidate of DE has the lowest OF among the three algorithms indicating superior searching ability of DE algorithm. As before, IA has a close UPQC location to DE because of limited and discrete searching space, but as for searching the best size of the UPQC, it has performed weaker compare to the DE. GA too has performed weaker in both finding the location and size of UPQC compared to DE and IA. 


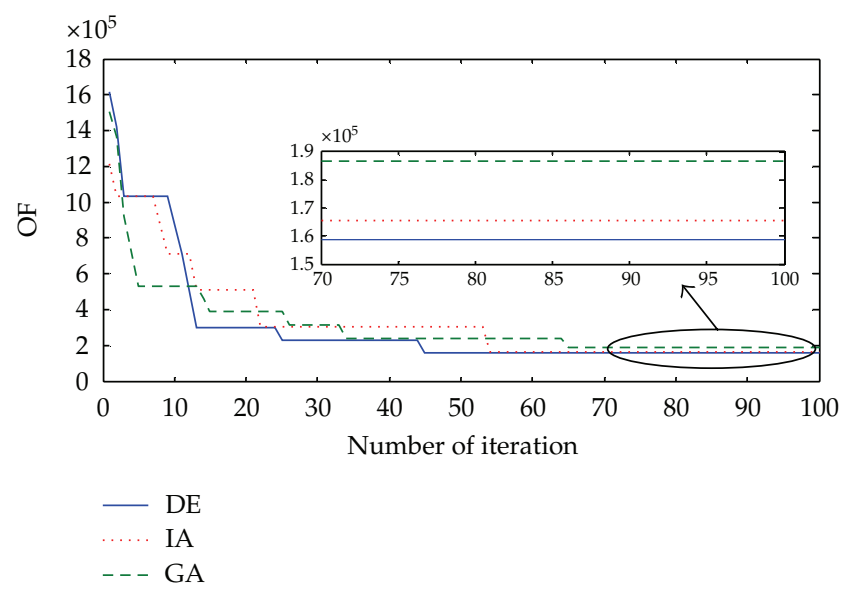

Figure 15: Convergence diagram of DE, IA, and GA methods.

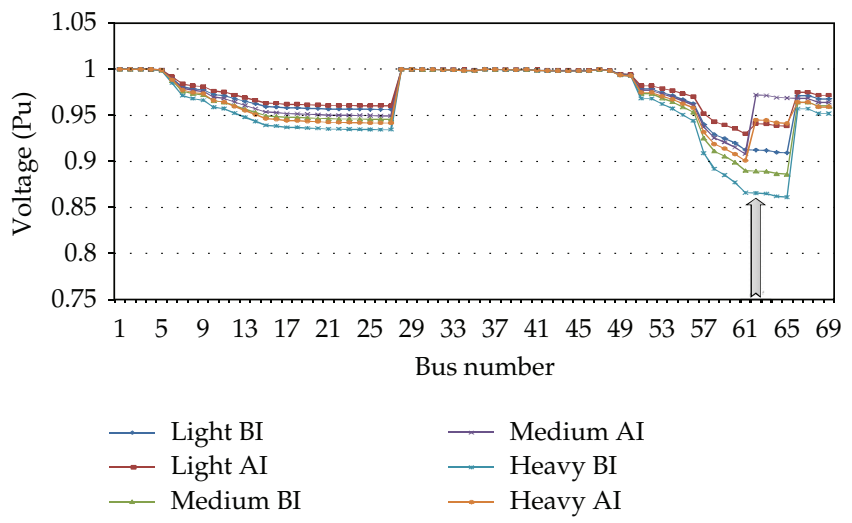

Figure 16: Voltages of the 69-bus distribution network before and after UPQC installation.

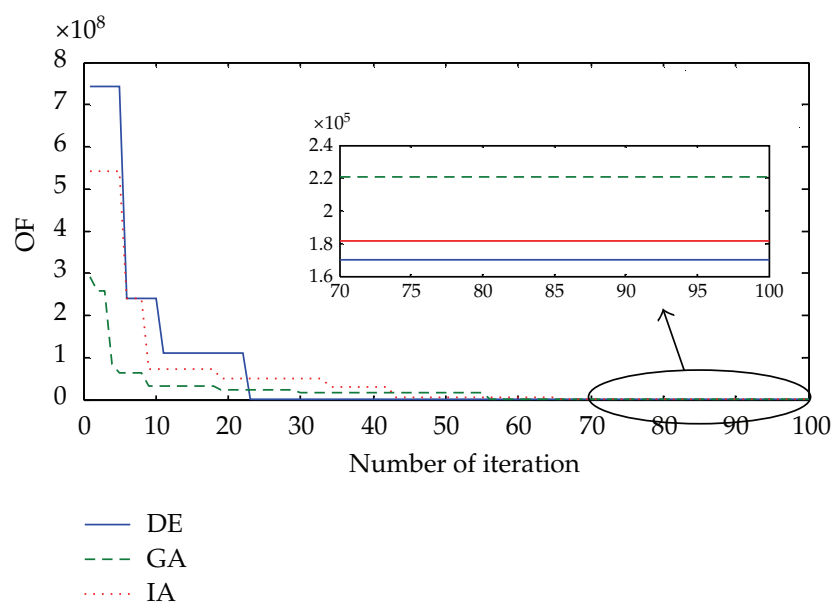

Figure 17: Convergence diagram of DE, IA, and GA methods. 


\section{Conclusion}

A new approach is proposed in this paper for the discrete optimization problem of UPQC placement and sizing in radial distribution systems using DE method. The DE method for minimizing continuous space functions has been introduced and shown to be superior to GA and IA methods. Energy and power losses due to installed UPQC as well as its associated cost are used to define the OF. For system solution, backward / forward sweep load flow is applied. Simulation results indicate that OF reduction may be obtained utilizing UPQC. Using DE method, the optimal location and size of UPQC is obtained in order to decrease power loss, cost of UPQC and current profile, and improve voltage. Compared with IA and GA, DE converges faster and smoother. Compared with GA and IA, DE technique provides minimum UPQC size, CPU time, and OF. Installation of the UPQC by the proposed approach leads to $18.2 \%$ and $21.42 \%$ power loss reductions, in 33- and 69-bus distribution systems, respectively. All bus voltages and currents of lines are within the desired boundaries. Reduction in total energy loss cost are $18.198 \%$ and $21.427 \%$ in 33- and 69-bus distribution network, respectively. Total cost saving as a result of this exercise is estimated to be of the order of $1.2 \%$ and $5.7 \%$ in the 33- and 69-bus IEEE test systems, respectively.

\section{References}

[1] A. Ghosh and G. Ledwich, "A unified power quality conditioner (UPQC) for simultaneous voltage and current compensation," Electric Power Systems Research, vol. 59, no. 1, pp. 55-63, 2001.

[2] R. C. Dugan, M. F. McGranaghan, and H. W. Beaty, Electrical Power System Quality, McGraw Hill, New York, NY, USA, 1996.

[3] E. Acha, V. G. Agelidis, O. Anaya-Lara, and T. J. E. Miller, Power Electronic Control in Electrical Systems, Newnes, Melbourne, Australia, 2002.

[4] A. Ghosh and G. Ledwich, "Compensation of distribution system voltage using DVR," IEEE Transactions on Power Delivery, vol. 17, no. 4, pp. 1030-1036, 2002.

[5] X. P. Zhang, "Advanced modeling of the multi-control functional static synchronous series compensator (SSSC) in Newton power flow," IEEE Transactions on Power Systems, vol. 18, no. 4, pp. 1410-1416, 2003.

[6] G. Chen, Y. Chen, L. F. Sanchez, and K. M. Smedley, "Unified power quality conditioner for distribution system without reference calculations," in Proceedings of the 4th International Power Electronics and Motion Control Conference (IPEMC '04), vol. 3, pp. 1201-1206, August 2004.

[7] A. K. Jindal, A. Ghosh, and A. Joshi, "Interline unified power quality conditioner," IEEE Transactions on Power Delivery, vol. 22, no. 1, pp. 364-372, 2007.

[8] H. Fujita and H. Akagi, "The unified power quality conditioner: the integration of series- and shuntactive filters," IEEE Transactions on Power Electronics, vol. 13, no. 2, pp. 315-322, 1998.

[9] F. Kamran and T. G. Habetler, "Combined deadbeat control of a series-parallel converter combination used as a universal power filter," IEEE Transactions on Power Electronics, vol. 13, no. 1, pp. 160-168, 1998.

[10] P. T. Nguyen and T. K. Saha, "Dynamic voltage restorer against balanced and unbalanced voltage sags: modelling and simulation," in Proceedings of the IEEE Power Engineering Society General Meeting, vol. 1, pp. 639-644, June 2004.

[11] M. Chawla, A. Rajvanshy, A. Ghosh, and A. Joshi, “Distribution bus voltage control using DVR under the supply frequency variations," in Proceedings of the IEEE Power India Conference, pp. 25-30, April 2006.

[12] M. Hu and H. Chen, "Modeling and controlling of unified power quality compensator," in Proceedings of the IEEE International Conference on Advances in Power System Control, Operation and Management, vol. 2, pp. 431-435, October 2000.

[13] Y. Y. Kolhatkar, R. R. Errabelli, and S. P. Das, "A sliding mode controller based optimum UPQC with minimum VA loading," in Proceedings of the IEEE Power Engineering Society General Meeting, pp. $2240-$ 2244, June 2005. 
[14] M. Basu, S. P. Das, and G. K. Dubey, "Performance study of UPQC-Q for load compensation and voltage sag mitigation," in Proceedings of the 28th Annual Conference of the IEEE Industrial Electronics Society, pp. 698-703, November 2002.

[15] F. Z. Peng, H. Akagi, and A. Nabae, "A new approach to harmonic compensation in power systems-a combined system of shunt passive and series active filters," IEEE Transactions on Industry Applications, vol. 26, no. 6, pp. 983-990, 1990.

[16] F. Silva, "Control methods for power converters," in Handbook of Power Electronics, M. H. Rashid, Ed., 2002.

[17] T. S. Key and J. S. Lai, “Comparison of standards and power supply design options for limiting harmonic distortion in power systems," IEEE Transactions on Industry Applications, vol. 29, no. 4, pp. 683-695, 1993.

[18] B. Han, B. Bae, H. Kim, and S. Baek, "Combined operation of unified power-quality conditioner with distributed generation," IEEE Transactions on Power Delivery, vol. 21, no. 1, pp. 330-338, 2006.

[19] G. Jianjun, X. Dianguo, L. Hankui, and G. G. Maozhong, “Unified power quality conditioner (UPQC): the principle, control and application," in Proceedings of the Power Conversion Conference, vol. 1, pp. 8085, 2002.

[20] C. Yunping, Z. Xiaoming, W. Jin, L. Huijin, S. Jianjun, and T. Honghai, "Unified power quality conditioner (UPQC): the theory, modeling and application," in Proceedings of the IEEE International Conference on Power System Technology, vol. 3, pp. 1329-1333, 2000.

[21] M. Hosseini, H. A. Shayanfar, and M. Fotuhi-Firuzabad, "Modeling of unified power quality conditioner (UPQC) in distribution systems load flow," Energy Conversion and Management, vol. 50, no. 6, pp. 1578-1585, 2009.

[22] V. Khadkikar, A. Chandra, A. O. Barry, and T. D. Nguyen, "Steady state power flow analysis of unified power quality conditioner (UPQC)," in Proceedings of the International Conference on Industrial Electronics and Control Applications (ICIECA '05), pp. 6-16, December 2005.

[23] R. Storn and K. Price, "Minimizing the real functions of the ICEC '96 contest by differential evolution," in Proceedings of the IEEE International Conference on Evolutionary Computation (ICEC '96), pp. 842-844, May 1996.

[24] R. Storn and K. Price, "Differential Evolution-a simple and efficient adaptive scheme for global optimization over continuous spaces," Journal of Global Optimization, vol. 11, no. TR-95-012, pp. 1-15, 1997.

[25] H. Akagi, "New trends in active filters for power conditioning," IEEE Transactions on Industry Applications, vol. 32, no. 6, pp. 1312-1322, 1996.

[26] M. Basu, S. P. Das, and G. K. Dubey, "Experimental investigation of performance of a single phase UPQC for voltage sensitive and non-linear loads," in Proceedings of the 4th IEEE International Conference on Power Electronics and Drive Systems, pp. 218-222, October 2001.

[27] R. Rajasree and S. Premalatha, "Unified power quality conditioner (UPQC) control using feed forward (FF)/ feed back (FB) controller," in Proceedings of the International Conference on Computer, Communication and Electrical Technology (ICCCET '11), pp. 364-369, March 2011.

[28] S. Ghosh and D. Das, "Method for load-flow solution of radial distribution networks," IEE Proceedings, vol. 146, no. 6, pp. 641-648, 1999.

[29] M. Hosseini, H. A. Shayanfar, and M. Fotuhi, "Modeling of series and shunt distribution facts devices in distribution load flow," Journal of Electrical Systems, vol. 4, no. 4, pp. 1-12, 2008.

[30] E. Acha, C. R. Fuerte-Esquivel, H. Ambriz-Perez, and C. Angeles-Camacho, FACTS Modeling and Simulation in Power Networks, Wiley, New York, NY, USA, 2004.

[31] I. C. da Silva Jr., S. Carneiro, E. J. de Oliveira, J. de Souza Costa, J. L. Rezende Pereira, and P. A. N. Garcia, "A heuristic constructive algorithm for capacitor placement on distribution systems," IEEE Transactions on Power Systems, vol. 23, no. 4, pp. 1619-1626, 2008.

[32] D. Das, "Optimal placement of capacitors in radial distribution system using a Fuzzy-GA method," International Journal of Electrical Power and Energy Systems, vol. 30, no. 6-7, pp. 361-367, 2008.

[33] S. A. Taher and M. K. Amooshahi, "Optimal placement of UPFC in power systems using immune algorithm," Simulation Modelling Practice and Theory, vol. 19, no. 5, pp. 1399-1412, 2011.

[34] S. A. Taher and A. A. Abrishami, "UPFC location and performance analysis in deregulated power systems," Mathematical Problems in Engineering, vol. 2009, Article ID 109501, 2009.

[35] D. Povh, "Modeling of FACTS in power system studies," in Proceedings of the IEEE Power Engineering Society Winter Meeting (PES '00), vol. 2, pp. 1435-1439, 2000. 
[36] M. Saravanan, S. M. R. Slochanal, P. Venkatesh, and J. P. S. Abraham, “Application of particle swarm optimization technique for optimal location of FACTS devices considering cost of installation and system loadability," Electric Power Systems Research, vol. 77, no. 3-4, pp. 276-283, 2007.

[37] R. Storn and K. Price, "Differential evolution-a simple and efficient adaptive scheme for global optimization over continuous spaces," ICSI Technical Report, no. TR-95-012, pp. 1-5, 1995.

[38] R. Storn and K. Price, "Differential evolution-a simple and efficient adaptive scheme for global optimization over continuous spaces," Journal of Global Optimization, vol. 11, no. 4, pp. 341-359, 1997.

[39] H. I. Shaheen, G. I. Rashed, and S. J. Cheng, "Optimal location and parameter setting of UPFC for enhancing power system security based on differential evolution algorithm," International Journal of Electrical Power and Energy Systems, vol. 33, no. 1, pp. 94-105, 2011.

[40] J. M. Ramirez, J. M. Gonzalez, and T. O. Ruben, "An investigation about the impact of the optimal reactive power dispatch solved by DE," International Journal of Electrical Power and Energy Systems, vol. 33, no. 2, pp. 236-244, 2011.

[41] S. A. Taher and M. Karim Amooshahi, “New approach for optimal UPFC placement using hybrid immune algorithm in electric power systems," International Journal of Electrical Power and Energy Systems, vol. 43, no. 1, pp. 899-909, 2012.

[42] A. A. Abou El Ela, M. A. Abido, and S. R. Spea, "Optimal power flow using differential evolution algorithm," Electric Power Systems Research, vol. 80, no. 7, pp. 878-885, 2010.

[43] A. Ketabi and M. J. Navardi, "Optimization shape of variable capacitance micromotor using differential evolution algorithm," Mathematical Problems in Engineering, vol. 2010, Article ID 909240, 15 pages, 2010.

[44] S. Sayah and K. Zehar, "Modified differential evolution algorithm for optimal power flow with nonsmooth cost functions," Energy Conversion and Management, vol. 49, no. 11, pp. 3036-3042, 2008.

[45] A. A. A. E. Ela, M. A. Abido, and S. R. Spea, "Differential evolution algorithm for optimal reactive power dispatch," Electric Power Systems Research, vol. 81, no. 2, pp. 458-464, 2011.

[46] M. Varadarajan and K. S. Swarup, "Network loss minimization with voltage security using differential evolution," Electric Power Systems Research, vol. 78, no. 5, pp. 815-823, 2008.

[47] I. Hussain and A. K. Roy, "Optimal size and location of distributed generations using differential evolution (DE)," in Proceedings of the 2 nd National Computational Intelligence and Signal Processing (CISP '12), pp. 57-61, 2012.

[48] C. Bulac, F. Ionescu, and M. Roscia, "Differential evolutionary algorithms in optimal distributed generation location," in Proceedings of the 14th International Conference on Harmonics and Quality of Power (ICHQP '10), pp. 1-5, September 2010.

[49] M. E. Baran and F. F. Wu, "Network reconfiguration in distribution systems for loss reduction and load balancing," IEEE Transactions on Power Delivery, vol. 4, no. 2, pp. 1401-1407, 1989.

[50] N. Mithulananthan, A. Sode-yome, and N. Acharya, "Application of FACTS controllers in Thailand power systems," FACTS project, Chulangkorn University, Final Report, 2005.

[51] K. Vijayakumar and R. P. Kumudinidevi, "A new method for optimal location of FACTS controllers using genetic algorithm," Journal of Theoretical and Applied Information Technology, vol. 3, no. 4, pp. 1-6, 2007.

[52] F. D. Galiana, K. Almeida, M. Toussaint et al., "Assessment and control of the impact of facts devices on power system performance," IEEE Transactions on Power Systems, vol. 11, no. 4, pp. 1931-1936, 1996.

[53] B. A. Renz, A. Keri, A. S. Mehraban et al., "AEP unified power flow controller performance," IEEE Transactions on Power Delivery, vol. 14, no. 4, pp. 1374-1381, 1999.

[54] T. A. Short, Electric Power Distribution Handbook, CRC Press, New York, NY, USA, 2004. 


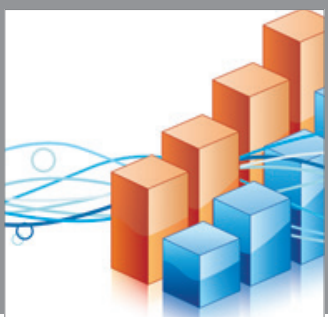

Advances in

Operations Research

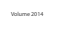

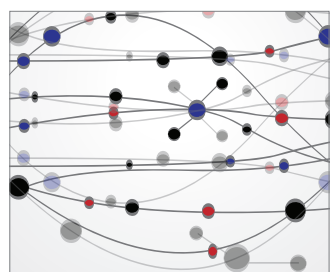

\section{The Scientific} World Journal
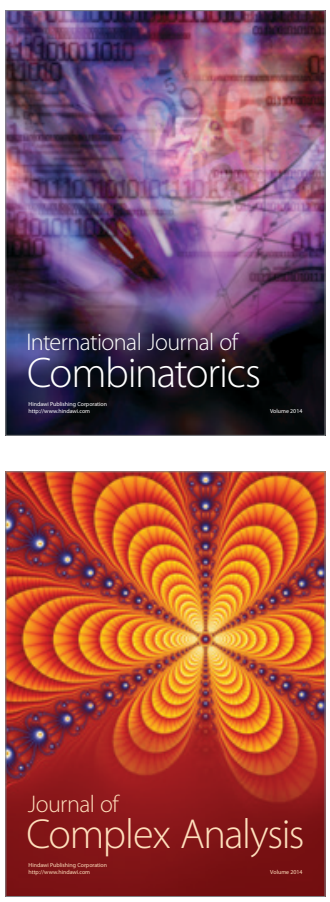

International Journal of

Mathematics and

Mathematical

Sciences
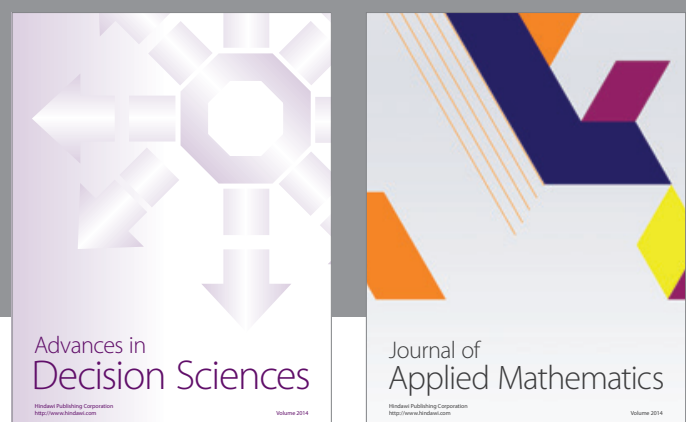

Journal of

Applied Mathematics
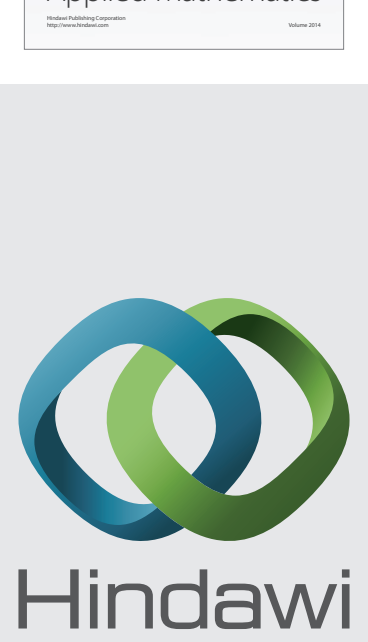

Submit your manuscripts at http://www.hindawi.com
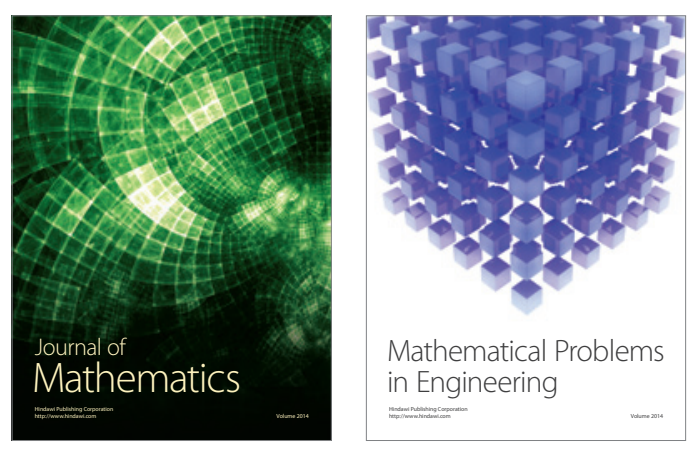

Mathematical Problems in Engineering
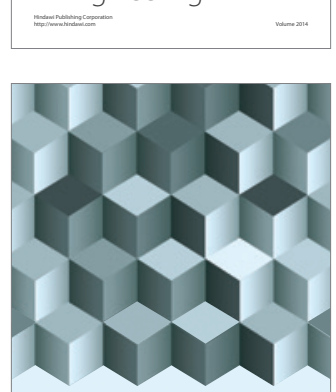

Journal of

Function Spaces
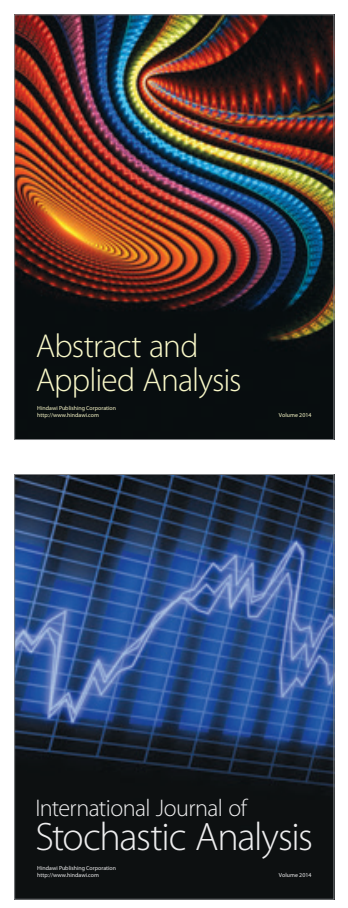

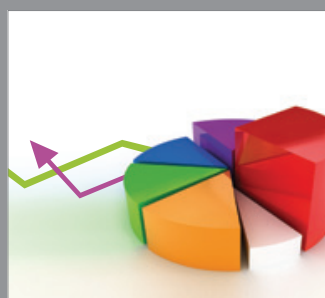

ournal of

Probability and Statistics

Promensencen
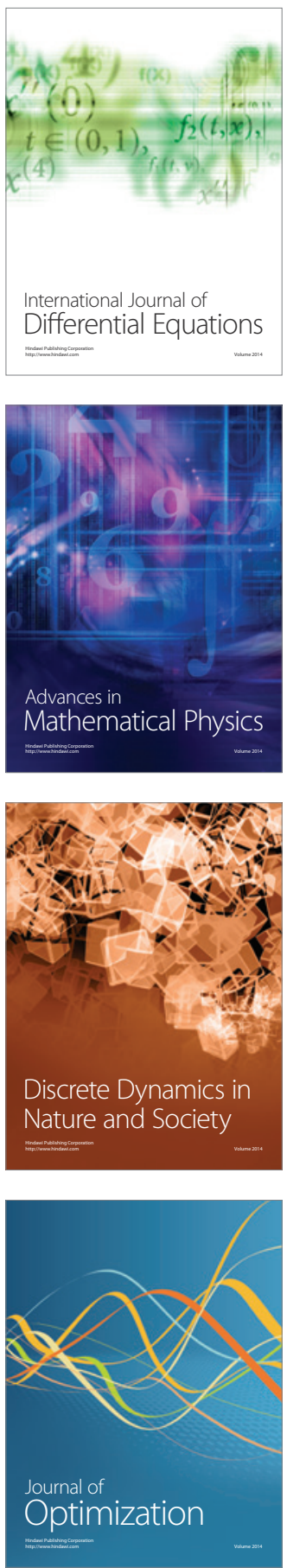